\title{
Utilización de plukenetia volubilis (sacha inchi) para mejorar los componentes nutricionales de la hamburguesa
}

\section{(Using plukenetia volubilis (sacha inchi) to improve the nutritional components of burger)}

\author{
Daniela Baldeón Clavijo", Francisco Velázquez Rodríguez¹, Jesús Eligio Castellanos \\ Estupiñán ${ }^{1}$
}

\begin{abstract}
Resumen:
Se evaluaron tres niveles consistentes en $10 \%, 15 \%$ y $20 \%$ de pasta de Plukenetia volubilis (Sacha Inchi) para sustituir al porcentaje en peso de grasa de cerdo utilizada convencionalmente a fin de mejorar la calidad nutricional de la hamburguesa común, comparada con un grupo de referencia. Las unidades experimentales fueron de 10 hamburguesas, con un peso de $100 \mathrm{~g}$ cada una y un total de 120. Se analizaron en un diseño completamente al azar, con tres repeticiones. La investigación se llevó a cabo en la Universidad Estatal Amazónica y los análisis bromatológicos y microbiológicos para determinar la calidad de la materia prima y los productos se realizaron en el laboratorio de la Facultad de Ciencias Químicas de la Universidad Central del Ecuador. Como complementos se realizaron pruebas sensoriales y estudios de Beneficio/Costo. Los resultados muestran a la variante de 10 $\%$ de pasta de Sacha Inchi como la más recomendable para el uso a nivel industrial.
\end{abstract}

Palabras clave: Plukenetia volubilis; Sacha Inchi; Hamburguesa; Alimentación funcional

\begin{abstract}
:
Three levels of paste Plukenetia volubilis (Sacha Inchi) consisting of $10,15 \%$ and $20 \%$ were evaluated to replace the weight percent lard conventionally used to improve the nutritional quality of the common hamburger, compared with a reference group. The experimental units were 10 burgers, weighing $100 \mathrm{~g}$. each and a total of 120 were analyzed in a completely randomized design with three replications. The research was conducted in the Universidad Estatal Amazónica and bromatológics and microbiological analyzes to determine the quality of the raw material and products are made in laboratory of the Faculty of Chemical Sciences of the Universidad Central del Ecuador. As supplements sensory tests and studies Benefit / Cost performed. The results show the variation of $10 \%$ pulp Sacha Inchi as the most recommended for use in industry.
\end{abstract}

Keywords: Plukenetia volubilis; Sacha Inchi; Burger; Functional food

\section{Introducción}

Se denominan "Alimentos Funcionales" (AF) aquellos que son elaborados no solo por sus propiedades nutritivas, sino también para cumplir una función específica como puede ser el mejorar la salud y reducir el riesgo de contraer enfermedades. Para ello se les agregan componentes biológicamente activos, como minerales, vitaminas, ácidos grasos, fibra alimenticia o antioxidantes, etc. A esta operación de añadir nutrientes exógenos se le denomina también fortificación. Entre los logros reconocidos en la literatura científica y en el marketing de los

\footnotetext{
1 Universidad Estatal Amazónica, Tena - Ecuador (dianasofiamc@gmail.com, \{fvelasquez, jestupinan\}@uea.edu.ec)
} 
productos alimenticios se encuentra la mejora de las funciones gastrointestinales, el aporte de sistemas redox y antioxidante, así como la modificación del metabolismo de macronutrientes (Roberfroid, M. 2000).

Los componentes más destacables de los alimentos funcionales son: la fibra dietética, los azúcares alcoholes o azúcares de baja energía, los aminoácidos, los ácidos grasos insaturados, los fitoesteroles, las vitaminas y los minerales, los antioxidantes, las bacterias ácido lácticas y otras sustancias excitantes o tranquilizantes (Salud, 2009).

La relación entre consumo de grasa y el desarrollo de enfermedades cardiovasculares ha generado que organizaciones como la ADA (American Dietetics Association, 1990) y la Organización Mundial de la Salud (FAO) (WHO, 1990), hayan modificado las recomendaciones dietéticas en la ingesta de grasa a menos del $30 \%$ de la ingesta calórica total y del colesterol en menos de 300 mg / día (Piñero, M. et al, 2008).

La emergente ciencia de los materiales alimentarios se está abocando al diseño de estructuras que sean apetecibles, saludables y dispensen nutrientes y compuestos bioactivos de manera efectiva durante la digestión (Aguilera, J. 2007). El interés del consumidor por la relación entre la dieta y la salud ha aumentado la demanda de información acerca de los alimentos funcionales.

Los componentes básicos de las grasas son los ácidos grasos. Se denominan ácidos grasos esenciales a aquellas grasas que no pueden ser sintetizadas o formadas por el organismo y por lo tanto es esencial que sean suministrados por la dieta. Estos compuestos son fundamentales para el transporte de distintos nutrientes, participan en los sistemas de defensa o inmunidad, son precursores de hormonas y ayudan a mantener las estructuras celulares. Los ácidos grasos omega se encuentran dentro de los denominados como esenciales por la razón de que el propio cuerpo humano no lo produce. Esto hace que deben ser ingeridos a través de una alimentación adecuada (Licata, M. 2009 b). Existen dos familias de ácidos grasos poliinsaturados esenciales: los "omega-6 y los omega-3". Su nombre omega-6 y omega-3 deriva de la configuración química del ácido graso, en este caso, de la ubicación del doble enlace en relación al metilo terminal $\left(\mathrm{CH}_{3}\right.$ terminal) (Castillo, C. 2009).

El fruto de la Plukenetia volubilis (Sacha Inchi) o maní del inca, es beneficioso para la salud, y es aconsejable incluirlo en las comidas ya que favorece el correcto desarrollo y funcionamiento, tanto del sistema nervioso como del cerebro pues es rico en ácidos grasos esenciales, como lo son los omega 3, omega 6 y omega 9 (Incainchi, 2009).

La materia prima oleaginosa que es la semilla de Sacha Inchi contiene altas cantidades de aceite (54\%) y relativamente alto contenido proteico (27\%) (Hamaker, E. et al., 1992). Por otro lado, García, H. (1992) reportó los siguientes resultados con respecto a la composición química del Sacha Inchi: proteína $24.22 \%$, humedad $5.63 \%$, grasa $43.10 \%$, carbohidratos $7.72 \%$ y ceniza 
2.80\%. Los estudios científicos actuales señalan a la Sacha Inchi como una de las mejores oleaginosas al ser comparada con algunas de uso clásico (maní, palma, soya, maíz, colza y girasol), por su composición y su alta calidad nutricional. El aceite tiene alto contenido en ácidos grasos esenciales omega 3 (más del 48\%) y omega 6 (36\%). Su digestibilidad es muy alta (más del $96 \%$ ) y contiene una elevado proporción de antioxidantes, vitamina A y vitamina $E$ (Agroindustrias Amazónicas, 2009). El contenido de otros compuestos como proteínas (33\%) y antioxidantes (50\%). demuestra su posibilidades de mejorar la alimentación al ser incluido en la dieta humana. Investigaciones recientes realizadas con aceites omegas y vitamina $E$ indican la importancia nutricional y terapéutica de su consumo para el control de radicales libres y una serie de enfermedades que estos originan en el organismo humano (Manco, C. 2006).

La producción del Sacha Inchi se inicia a los 6,5 meses del trasplante, obteniéndose en el primer año rendimientos promedios de 0,7 a 2,0 t/ha. Se desarrolla en asociación y con cultivos de cobertura, alcanzando edades hasta de 10 años.

La Organización Mundial de la Salud (OMS) afirma en sus estudios (OMS, 2009) que el mundo desarrollado consume más del doble de los requerimientos diarios necesarios en proteínas mientras el tercer mundo sufre una carencia alarmante de proteínas. Por otro lado, la propia OMS recomienda una dieta con proporción de sólo el 25 \% de proteína animal sustituyendo el otro 75 \% con proteína de origen vegetal. La administración proteica en una dieta debe ser constante. Desde el punto de vista cuantitativo, las proteínas aportan 4 Kcal por gramo, y la recomendación es que su consumo sea de 1 gramo de proteína por kg de peso del consumidor (Licata, M. 2009 a).

Se considera que el valor nutritivo de las proteínas de la carne es superior al de las proteínas vegetales. Para la nutrición humana es tan importante la calidad como la cantidad de las proteínas ingeridas. Tanto el hombre como los animales solo pueden sintetizar parte de los aminoácidos que necesitan para la construcción de sus propias proteínas, los otros, denominados aminoácidos esenciales, tienen que ser necesariamente suministrados por la dieta (Prandl, O. et al, 1994).

Las culturas alimenticias en los países Latinoamericanos se han transformado por la influencia de los países más ricos. La transnacional McDonald ha llevado por todo el mundo las hamburguesas y este hecho ha estimulado a que muchos países hayan transformado sus hábitos alimentarios, imponiéndose el uso de las comidas rápidas. En definitiva, el alto consumo de hamburguesas en numerosos países no es otra cosa que el resultado de la toma de conciencia de un nuevo modo de alimentarse que sigue criterios más racionales, lógicos y económicos. Como un producto rico en proteínas de alta calidad, vitaminas y otros compuestos fácilmente asimilables (Barreda, $\mathrm{P}$. 2009), se considera una de las favoritas en las comidas rápidas. Por esta razón es siempre recomendable adicionarle algún ingrediente que pueda aportar un beneficio para la salud y que contribuya al equilibrio del colesterol, principal causa de mortalidad en el mundo. 
El descubrimiento científico de las propiedades de la Sacha Inchi o maní de los incas ha elevado esta planta amazónica a un primerísimo lugar para la alimentación y la medicina tradicional al constituir una de las fuentes vegetales más grandes de omega, un ácido graso esencial para la vida humana (Sacha Inchi, 2007). La composición en ácidos grasos del aceite crudo determinada por cromatografía de gases reveló un alto grado de instauración (90,34 \%) destacándose el ácido linolénico (43,75 \%) seguido del ácido linoleico con 36,99 \%. Entre los ácidos grasos saturados el más significativo fue el ácido palmítico con 5.61 \% (Pascual, G. y Mejía, L. 2009). En comparación a los aceites de todas las semillas oleaginosas utilizadas en el mundo, para consumo humano, el Sacha Inchi es el más rico en ácidos grasos insaturados, llega hasta 93,6 \% (Laboratorio vida nueva, 2009).

El presente trabajo persigue como objetivo evaluar la factibilidad técnica de la utilización de Plukenetia volubilis (Sacha Inchi) para mejorar los componentes nutricionales de la hamburguesa.

\section{Materiales y Métodos}

La hamburguesa es un producto molido, fresco que se prepara con carne de diferentes especies entre ellas la de res, mezclada con grasa de cerdo y aumentado con harinas y/o almidones (yuca, papa) y que debe ser congelada para su conservación. Las hamburguesas tienen un picado grueso para producir textura fibrosa y desmenuzable, idealmente la acción mecánica, junto con el cloruro de sodio, debería ser suficiente para ligar el producto antes y después del cocinado. En la práctica la necesidad de romper el tejido conectivo hace que sea necesario un picado más intenso. El uso de carne de baja calidad, que contenga grandes cantidades de tejido conectivo puede hacer que la hamburguesa tenga una textura no deseable, muy cohesiva. (Varnam, A. y Sutherland, J. 1995). Venegas, G. y Piñeros, G. (2002), describen que la pasta de hamburguesa se hace mezclando carnes y grasa, y permite el uso de harina de trigo y agua, así como la adición de especias frescas, como cilantro, cebolla larga o cabezona, apio y perejil, pimentón, ajo, yerbabuena y otras.

El picado reduce los problemas de obstrucción por la grasa y el tejido conjuntivo lo que también incide favorablemente sobre las propiedades de la cocción. El tipo más común de picado se consigue con un molino de tornillo que funciona en una cámara horizontal. Las piezas de carne se introducen desde un extremo del molino, y es recomendable no usar carne congelada. Habitualmente en el proceso se produce un aumento de temperatura de hasta $10 \stackrel{\circ}{ } \mathrm{C}$. Algunos tipos de molino permiten que se use carne semicongelada y en ese caso, el calor latente de fusión limita el aumento de temperatura. La parte externa de la salida consiste en una placa perforada fija, que está situada adyacente a una cuchilla rotatoria o una placa perforada rotatoria. Las picadoras producen un picado relativamente grueso con partículas irregulares debido a la acción de las rasgaduras. 
La presente investigación se desarrolló en la Planta Piloto de Procesos Agroindustriales de la Universidad Estatal Amazónica, ubicada en la ciudad de Puyo en el kilómetro 2,5 de la vía Tena, cantón Pastaza, provincia de Pastaza, Ecuador y tuvo una duración de 120 días. Las condiciones meteorológicas imperantes en la zona se describen en Tabla 1. En la misma se destacan la baja temperatura promedio, la elevada humedad relativa y la alta tendencia a la lluvia que existe en la región de Pastaza.

Tabla 1. Condiciones meteorológicas de la Ciudad de Puyo, Cantón Pastaza

\begin{tabular}{|l|l|}
\hline PARAMETRO & DATOS \\
\hline Altitud $(\mathrm{msnm})$ & 900 \\
\hline Temperatura $\left({ }^{\circ} \mathrm{C}\right)$ & 21 \\
\hline Humedad relativa (\%) & $87-89$ \\
\hline Pluviosidad (mm) & 3500 \\
\hline
\end{tabular}

Fuente: Aeropuerto "Río Amazonas", 2014

Previo al inicio del experimento se realizaron pruebas en la elaboración de la hamburguesa, en las cuales se analizó el efecto de añadir a la hamburguesa 10, 15, 20 y $30 \%$ de Sacha Inchi. La muestras con 10, 15 y $20 \%$ de adición permitió la liga de los ingredientes de la hamburguesa y con $30 \%$ de adición no se logró una liga adecuada de la mezcla. Por tal motivo se utilizaron únicamente los niveles 10, 15 y $20 \%$ de adición de Sacha Inchi para la fabricación de la hamburguesa. La unidad experimental en la presente investigación estuvo constituida por 10 hamburguesas con un peso de $100 \mathrm{~g}$ cada una, siendo necesarias un total de 120 hamburguesas para el experimento. Se evaluaron los tres niveles de utilización de pasta de Plukenetia volubilis (10, 15 y $20 \%$ ), en sustitución al porcentaje en peso de la grasa de cerdo utilizada convencionalmente en la elaboración de hamburguesas, los resultados fueron comparados con un grupo de Referencia, en el cual no se incluyó la pasta. El esquema del experimento empleado se especifica en la Tabla 2.

Tabla 2. Esquema del experimento

\begin{tabular}{|l|c|c|c|c|}
\hline \multicolumn{1}{|c|}{ Tratamientos } & Código & T.U.E. & Réplicas & Hamburguesa/Tratamiento \\
\hline $0 \%$ Sacha Inchi & $\mathrm{R}$ & 10 & 3 & 30 \\
\hline $10 \%$ Sacha Inchi & SI10 & 10 & 3 & 30 \\
\hline $15 \%$ Sacha Inchi & SI15 & 10 & 3 & 30 \\
\hline $20 \%$ Sacha Inchi & SI20 & 10 & 3 & 30 \\
\hline Total & & & & 120 \\
\hline
\end{tabular}

T.U.E.: Tamaño de Unidad Experimental

Para la distribución de los tratamientos se utilizó el Diseño Completamente al Azar. Los resultados experimentales fueron sometidos a un Análisis de Varianza (ADEVA) con separación 
de medias por el método de rango múltiple de Tukey a un nivel de significación de 0,05 y análisis de estadística no paramétrica (Prueba de Kruskal Wallis).

Las variables estudiadas en esta investigación fueron las siguientes:

1. Características Bromatológicas (Humedad, Materia seca, Proteína, Grasa, Fibra, Carbohidratos, Ceniza)

2. Contenido de Ácidos grasos (Palmítico, Esteárico, Mirístico, Láurico, Oleico, Linoleico, Palmitoleico, Alfa linolénico, Araquídico)

3. Análisis Microbiológico (Recuentos de Mohos y Levaduras, Recuentos de Coliformes Totales, Escherichia coli, Stafilococcus aureus, Salmonella)

4. Análisis Sensorial (Aceptabilidad, Intención de compra)

5. Análisis Económico (Ingresos y egresos, Beneficio/Costo)

Los análisis se realizaron en el laboratorio de la Facultad de Ciencias Químicas de la Universidad Central del Ecuador. Para los análisis bromatológicos se entregó una muestra de hamburguesa cocida debidamente guardada en una funda plástica marca "Reynolds" (bolsas resellables medianas para congelar), transportada en cooler manteniéndola a una temperatura de $8 \stackrel{\circ}{ } \mathrm{C}$ para todos los tratamientos. La muestra de maní tostado (Sacha Inchi) no requirió de refrigeración. Estas muestras fueron analizadas para cada parámetro con un método específico como se describe en la Tabla 3.

Tabla 3. Métodos analíticos usados para los análisis bromatológicos

\begin{tabular}{|l|c|}
\hline Parámetro & Método \\
\hline Proteína & MAL-04 \\
\hline Grasa & 39.1.19 Método oficial AOAC 981.10 \\
\hline Humedad & MAL-03 \\
\hline Cenizas & MAL-13 Método oficial AOAC 991.36 \\
\hline Fibra & 33.1.03 Método oficial AOAC 925.10 \\
\hline Carbohidratos & MAL-02 \\
\hline Calorías & MAL-50 \\
\hline
\end{tabular}


Para los análisis microbiológicos las muestras se entregaron al mismo laboratorio en similares condiciones de conservación. Estas muestras fueron analizadas para cada parámetro con los métodos que se describe en la Tabla 4. Los análisis se complementan con estudios de aceptabilidad e intención de compra. Finalmente se realiza un análisis de Beneficio/Costo para determinar la factibilidad económica de la propuesta.

Tabla 4. Métodos usados para el análisis microbiológico de la hamburguesa cruda

\begin{tabular}{|l|c|}
\hline Parámetros & Métodos \\
\hline Recuentos de mohos & AOAC 997.02 \\
\hline Recuentos de levaduras & AOAC 997.02 \\
\hline Recuentos de Coliformes Totales & AOAC 991.14 \\
\hline Escherichia coli (recuento) & AOAC 991.14 \\
\hline Stafilococcus aureus (recuento) & AOAC 2003.11 \\
\hline Salmonella spp. (Identificación/25g) & NTE INEN 1529-15:96 \\
\hline
\end{tabular}

En la Figura 1 se muestra la secuencia de preparación de la pasta de Sacha Inchi. Obsérvese como se detallan las temperaturas y el tiempo de operación en los casos que se requieren.

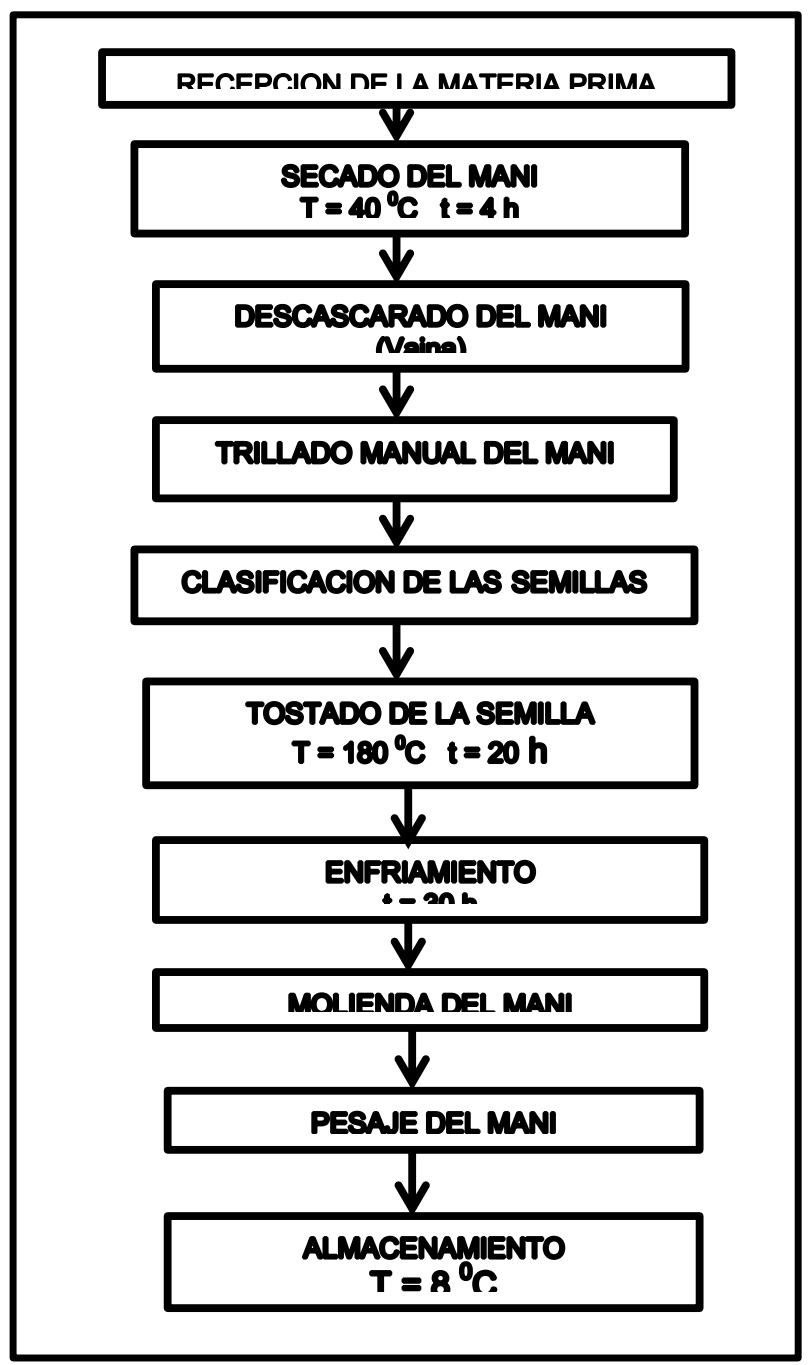

Figura 1. Diagrama de flujo del proceso de producción de la pasta de Sacha Inchi 
En la Tabla 5 se presentan las composiciones de la materia prima usada para la formulación de las hamburguesas sometidas a investigación. En la misma se observa que se mantienen invariables los valores porcentuales de carne de res y harina de trigo y como se van sustituyendo las proporciones de grasa de cerdo por partes proporcionales de pasta de Plukenetia volubilis.

Tabla 5. Materiales usados para la formulación de hamburguesa

\begin{tabular}{|l|c|c|c|c|}
\hline Ingredientes & $\mathbf{R}(\%)$ & $\mathbf{T}_{\mathbf{1}}(\mathbf{\%})$ & $\mathbf{T}_{\mathbf{2}}(\mathbf{\%})$ & $\mathbf{T}_{\mathbf{3}}(\%)$ \\
\hline Carne de res & 70 & 70 & 70 & 70 \\
\hline Grasa de cerdo & 20 & 10 & 5 & 0 \\
\hline Pasta de Sacha Inchi & 0 & 10 & 15 & 20 \\
\hline Harina de trigo & 10 & 10 & 10 & 10 \\
\hline
\end{tabular}

La composición de las hamburguesas se complementa con la adición de aditivos y condimentos. La Tabla 6 muestra los porcentajes de aditivos y condimentos usados en la formulación.

Tabla 6. Aditivos y condimentos usados para la formulación de la hamburguesa

\begin{tabular}{|l|c|}
\hline Aditivos y condimentos & Porcentaje \\
\hline Agua & 15,000 \\
\hline Nitratos & 0,015 \\
\hline Sal & 2,300 \\
\hline Tripolifosfatos & 0,170 \\
\hline Ácido Ascórbico & 0,015 \\
\hline Cebolla perla fresca & 3,330 \\
\hline Pimiento fresco & 2,000 \\
\hline Ajo fresco & 2,000 \\
\hline
\end{tabular}

En la Figura 2 se muestra la secuencia tecnológica del proceso de elaboración de la hamburguesa. En la misma aparecen las propiedades en cada etapa del proceso, fundamentalmente las temperaturas inicial y final y los tiempos de operación recomendados.

\section{Resultados y Discusión}

La Tabla 7 muestra la caracterización química de la Sacha Inchi de acuerdo a las técnicas analíticas especificadas en la Tabla 3. Los dos primeros índices son el contenido de agua y sólidos de la semilla. A partir de ahí se relacionan los componentes del sólido divididos en Proteína, Grasa Cruda, Fibra Cruda, Cenizas y Carbohidratos. Finalmente se expone el contenido energético de la almendra descascarada y tostada.

El contenido de humedad de 2,60 \% se encuentra por debajo de los resultados reportados por García, H. (1992) quien determinó un promedio de 5,63 \% y Sacha Inchi Corporation y LRF 
Representaciones S.A.C (2009) un promedio de 6,37\%. Este hecho guarda relación con el alto contenido de materia seca $(97,40 \%)$, que supera en un $3,03 \%$ a los valores reportados por dicho autor.

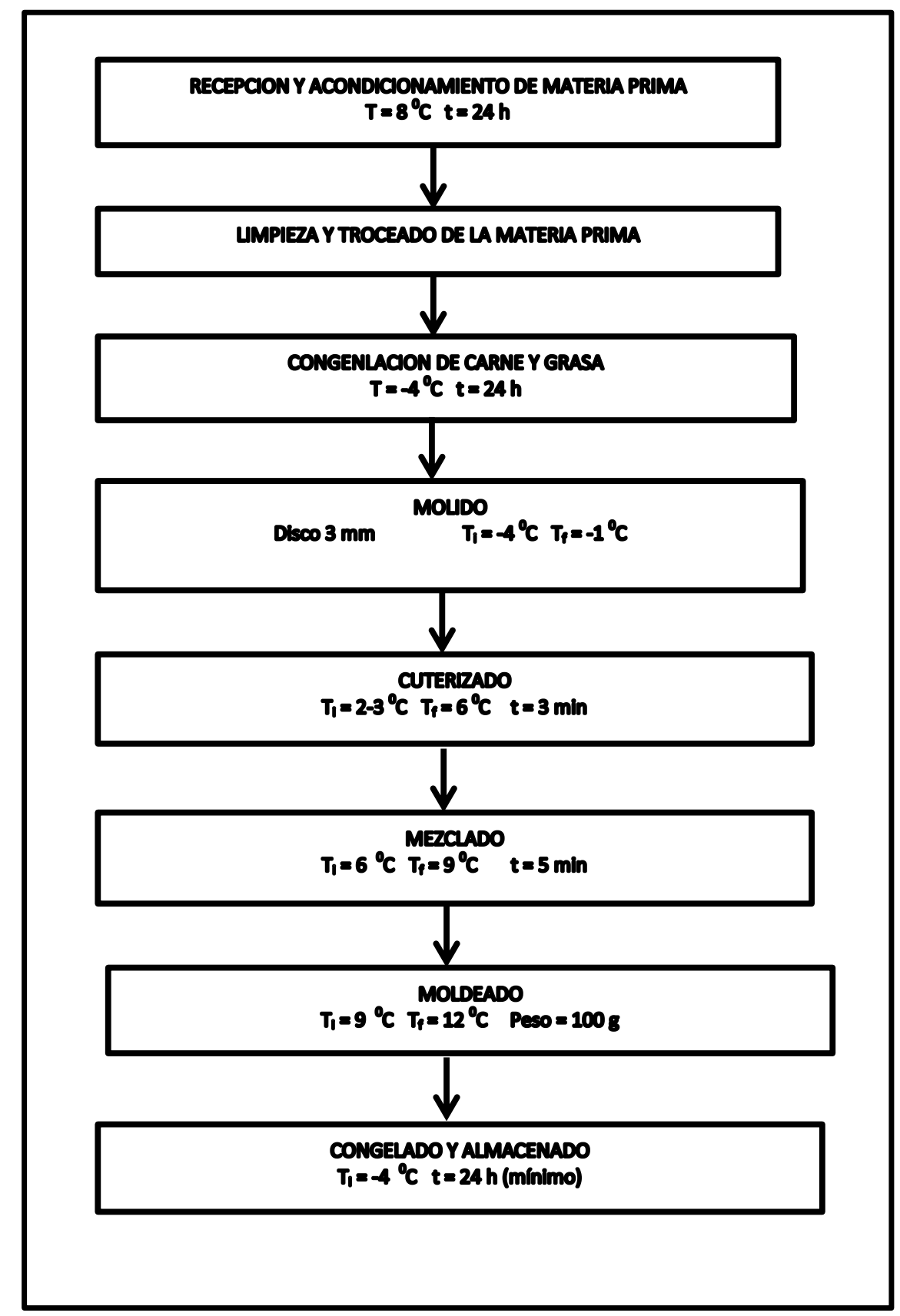

Figura 2. Diagrama de flujo del proceso de elaboración de la hamburguesa

El contenido de proteína del Plukenetia volubilis (Sacha Inchi) alcanzó un promedio de 28,50 \%. Este valor es similar al reportado por Sacha Inchi Corporation y LRF Representaciones S.A.C (2009), con un $28,52 \%$ de proteínas en la almendra, Hamaker, E. et al, (1992), determinaron un contenido de proteína de 27,00 \%. Por otro lado Obregón, L. (1993), manifiesta que el Plukenetia volubilis contiene $23,00 \%$ de proteína ofreciendo perspectivas para su uso. Así mismo García, H. (1992) determinó un contenido de 24,22 \% de proteína en el Plukenetia volubilis. Por lo 
anteriormente expuesto el alto contenido de proteína de esta leguminosa puede ser aprovechado en la industria cárnica para la elaboración de productos.

Tabla 7. Caracterización química del Sacha Inchi Amazónico Ecuatoriano

\begin{tabular}{|l|c|}
\hline Componente & Resultado \\
\hline Humedad (\%) & 2,6 \\
\hline Materia seca (\%) & 94,4 \\
\hline Proteína (\%) & 28,50 \\
\hline Grasa Cruda (\%) & 52,63 \\
\hline Fibra Cruda (\%) & 1,02 \\
\hline Cenizas (\%) & 2,80 \\
\hline Carbohidratos (\%) & 12,44 \\
\hline Calorías (Kcal/100 g) & 637,4 \\
\hline
\end{tabular}

El contenido de la grasa cruda determinada en el Plukenetia volubilis fue de 52,63\%. Al respecto, Hazan y Stovesand (1980) reportaron un 54 \%, Hamaker, E. et al (1992) un 49 \%, Obregón, L. (1993) el 49,9 \%, Pascual, G. y Mejía, L. (2009) un promedio de 51,4 \% de grasa cruda en el grano descascarado y tostado. Como puede observarse estos valores no difieren mucho al compararlos con los reportados por los especialistas mencionados.

La fibra cruda determinada en el producto utilizado alcanzó un contenido de 1,02 \%. Este resultado difiere con lo reportado por Sacha Inchi Corporation y LRF Representaciones S.A.C (2009), que es de un 2,6\% de fibra.

El indicador de ceniza presentó un valor de 2,8 \%, similar al reportado por Sacha Inchi Corporation y LRF Representaciones S.A.C (2009) que fue de 2,1\%.

El contenido de carbohidratos encontrado en la investigación fue de 12,44\%, intermedio a lo indicado por Sacha Inchi Corporation y LRF Representaciones S.A.C (2009) que reporta un porcentaje de carbohidratos entre $17,7 \%$ y $7,72 \%$.

La energía calórica del Plukenetia volubilis reportado en el ensayo fue de 637,4 Kcal/100g, superando a los resultados reportados por Sacha Inchi Corporation y LRF Representaciones S.A.C (2009) con 555,7 Kcal/100g, y otros investigadores con $562 \mathrm{Kcal} / 100 \mathrm{~g}$ respectivamente. Este resultado sugiere que el Sacha Inchi amazónico ecuatoriano constituye un alimento con mayor potencial calórico que los productos a los que se refieren las fuentes bibliográficas.

En los análisis realizados a la semilla de Plukenetia volubilis se encontraron los ácidos grasos saturados: Palmitico (3,84 \%) y Esteárico (2,41\%), mientras que el contenido de ácidos grasos insaturados fue de 10,28 \% de Oléico (Omega 9), 35,69 \% de ácido Linoléico (Omega 6) y 47,74 
\% de ácido Alfa linolénico (Omega 3). De los ácidos Palmitoléico y Araquídico solo se determinaron trazas.

Los resultados obtenidos demuestran que el Sacha Inchi es un alimento funcional que proporciona beneficios para la salud más allá de la nutrición básica, coincidiendo con lo expresado por Vázquez, M. (2005). Su consumo le da energía al cerebro, limpia el torrente sanguíneo, y lleva los nutrientes a las células (Anaya, 2006). El contenido de ácidos grasos en el Sacha Inchi es similar a lo reportado por Inca Inchi (2009): alfa linolénico 48,60 \%, ácido linoléico 36,80 \% y ácido oleico $8,28 \%$.

Los resultados del análisis bromatológico realizado a las hamburguesas se exponen en la Tabla 8. El contenido de humedad en los diferentes tipos de hamburguesas cocidas presentó diferencias estadísticas $(P<0,01)$, es así que las hamburguesas del grupo Control y del $10 \%$ de Sacha Inchi, presentaron los mayores contenidos de humedad, alcanzando promedios de 58,93 y 58,86\% respectivamente, seguidos por el contenido de humedad en las hamburguesas pertenecientes al tratamiento de $15 \%$ de Sacha Inchi que alcanzaron el 54,53\%, en tanto que el menor contenido de humedad se registró en el grupo de hamburguesas elaboradas con 20 \% de pasta de Sacha Inchi, en el cual se determinó un 54,09 \% de humedad. Los resultados anteriormente descritos para esta variable están relacionados a la capacidad de retención de humedad que tienen las hamburguesas de cada tratamiento. Además Ramos, M. (2008), manifiesta que el agua representa el $75 \%$ del peso total de la carne y únicamente el $5 \%$ del total de agua favorece a la unión de los puentes de hidrógeno con los grupos hidrófilos cargados de proteína.

Tabla 8. Composición química de los cuatro tipos de hamburguesas cocidas mediante el empleo de diferentes niveles de Sacha Inchi

\begin{tabular}{|c|c|c|c|c|c|c|}
\hline \multicolumn{7}{|c|}{ Niveles de Sacha Inchi } \\
\hline Parámetro & 0 & 10 & 15 & 20 & Probabilidad & $\% \mathbf{C V}$ \\
\hline Humedad \% & $58,93 a$ & $58,86 a$ & $54,53 b$ & $54,09 b$ & $0,0001^{* *}$ & 0,31 \\
\hline Materia Seca \% & $41,07 b$ & $41,14 b$ & $45,47 a$ & $45,90 a$ & $0,0001^{* *}$ & 0,41 \\
\hline Proteína \% & $18,57 \mathrm{c}$ & $20,86 a$ & $20,31 b$ & $20,42 b$ & $0,0001^{* *}$ & 0,31 \\
\hline Grasa Cruda \% & $13,35 a$ & $12,91 b$ & $11,90 \mathrm{c}$ & $9,84 d$ & $0,0001^{* *}$ & 0,86 \\
\hline Fibra Cruda \% & $0,21 c$ & $0,28 b$ & $0,19 c$ & $0,32 a$ & $0,0001^{* *}$ & 5,34 \\
\hline Cenizas \% & $2,98 d$ & $3,23 a$ & $3,17 b$ & $3,04 c$ & $0,0001^{* *}$ & 0,48 \\
\hline Carbohidratos \% & $5,80 \mathrm{c}$ & $8,20 \mathrm{a}$ & $8,44 a$ & $7,46 b$ & $0,0001^{* *}$ & 2,45 \\
\hline Calorías Kcal/100g & 217,6 & 234,4 & 222,4 & 200,0 & - & - \\
\hline
\end{tabular}

Letras iguales no difieren estadísticamente según Turkey $(\mathrm{P}<0.05)$

\% CV: Porcentaje de Coeficiente de Variación

**: Diferencia altamente significativa entre promedios

La materia seca en los diferentes tipos de hamburguesas cocidas presentó diferencias estadísticas $(P<0,01)$ obteniéndose el mayor valor en las hamburguesas de los tratamientos 15 y 
$20 \%$ de pasta de Sacha Inchi con 45,47 y 45,90 \% de materia seca, seguido por el contenido de materia seca determinado en las hamburguesas de los tratamientos $10 \%$ de Sacha Inchi y Testigo con promedios de $41,14 \%$ y $41,07 \%$ respectivamente. Estos valores presentan correspondencia con los valores de humedad.

El contenido de proteína en los diferentes tipos de hamburguesa cocida presentó diferencias significativas $(P<0.01)$, es así que las hamburguesas elaboradas con $10 \%$ de pasta de Sacha Inchi con 20,86\% obtuvo el mayor promedio, posteriormente las hamburguesas elaboradas con 15 y $20 \%$ de Sacha Inchi no presentan significación entre si, presentando valores de 20,31 y 20,42 $\%$ respectivamente, pero si existen diferencias al compararlos con el contenido de proteína determinado en las hamburguesas pertenecientes al grupo control que alcanzaron un promedio de $18,57 \%$, valor similar al obtenido por Melgarejo y Maury (2002) con 18,90\%.

El aumento en el porcentaje de proteína con respecto a la referencia se debe a la inclusión de pasta de Sacha Inchi (28,50\% de proteína). Al mezclarse entre sí las proteínas de origen animal y las de origen vegetal mejoran la hamburguesa convirtiéndola en un producto funcional. Una respuesta normal es la que se produce en los tratamientos 15 y $20 \%$ de pasta de Sacha Inchi que al no contener grasa de cerdo (mejor emulsionante de los embutidos Salazar (2008)), la emulsión se rompe al momento de la cocción, perdiéndose el valor nutritivo de varios ingredientes, en este caso de proteína.

Los resultados obtenidos con respecto al contenido de grasa cruda en los diferentes tipos de hamburguesa cocida demuestran que mientras más pasta de Sacha Inchi se utiliza en la hamburguesa, será menor el contenido de grasa. Así los valores obtenidos alcanzan 13,35 \% de grasa para las hamburguesas del tratamiento control, 12,91\% para las hamburguesas elaboradas con $10 \%$ de Sacha Inchi, 11,90\% de grasa en las hamburguesas elaboradas con $15 \%$ de pasta de Sacha Inchi y finalmente $9.84 \%$ de grasa en las hamburguesas elaboradas con $20 \%$ de pasta de Sacha Inchi, presentando diferencias significativas $(P<0.01)$, entre todos los tratamientos. Estos resultados se encuentran en correspondencia al contenido de la grasa de cerdo y del Sacha Inchi (100\% de grasa en de cerdo), 52,63 \% de grasa en el Sacha Inchi).

La disminución en el contenido de grasa con la adición de Sacha Inchi en las hamburguesas puede tener consecuencias favorables a nivel nutricional, pues el consumo de dietas con alto contenidos de grasas, especialmente saturadas, tiene consecuencias negativas para la salud humana, y la reducción de éstas, es una de las tendencias más fuertes en el desarrollo de los productos alimenticios de hoy (Yánez, E. y Biolley, E. 1999).

El contenido de fibra en los diferentes tipos de hamburguesa cocidas presentó diferencias estadísticas $(P<0.01)$, obteniendo valores de $0,32 \%$ para las hamburguesas elaboradas con $20 \%$ de Sacha Inchi, seguido por las hamburguesas procesadas con $10 \%$ de pasta de Sacha Inchi con 
0,28 \%, mientras que las hamburguesas de los tratamientos Control y $15 \%$ de adición de Sacha Inchi alcanzaron valores de 0,21 y 0,19 \% respectivamente, los cuales no tienen significación entre ellos, pero si con el resto de los tratamientos. La fibra no es un componente primordial de la hamburguesa, los resultados obtenidos en la investigación se consideran bajos al compararse con los reportados que han sido de $1,96 \mathrm{~g} / 100 \mathrm{~g}$.

El contenido de cenizas en los diferentes tipos de hamburguesa cocidas presentó diferencias estadísticas $(P<0,01)$, obteniéndose el mayor valor en las hamburguesas del tratamiento $10 \%$ de Sacha Inchi con 3,23 \%, seguido por el contenido de ceniza en las hamburguesas del tratamiento $15 \%$ de pasta de Plukenetia volubilis con 3,17\%, luego por las cenizas contenidas en las hamburguesas del tratamiento $20 \%$ de Sacha Inchi con un valor de 3,04\%, y finalmente con el menor contenido de ceniza fue determinado en las hamburguesas del tratamiento testigo con 2,98 $\%$.

En lo referente al contenido de carbohidratos en los diferentes tipos de hamburguesa cocidas existe diferencias estadísticas $(P<0.01)$, es así que las hamburguesas de los tratamientos 10 y 15 $\%$ de Plukenetia volubilis presentaron los mayores contenidos de carbohidratos, alcanzando promedios de $8,20 \%$ a $8,44 \%$ respectivamente, seguidos por el contenido de carbohidratos en las hamburguesas elaboradas con $20 \%$ de Sacha Inchi que alcanzaron un promedio de 7,46 \%, en tanto que el menor contenido de carbohidratos se registró en el grupo de hamburguesas del grupo Control, en el cual se determinó un 5,80 \%. En el caso de las hamburguesas elaboradas con $20 \%$ de Sacha Inchi, el contenido disminuye por perdida de ingredientes en la cocción, debido a que la hamburguesa rompe su emulsión.

Los valores de calorías calculados para los diferentes tipos de hamburguesa cocidas son: para el tratamiento Control un valor de $217,6 \mathrm{Kcal} / 100 \mathrm{~g}$, para las hamburguesas del tratamiento $10 \%$ de Sacha Inchi $234,4 \mathrm{Kcal} / 100 \mathrm{~g}$, luego en las hamburguesas del tratamiento $15 \%$ de pasta de Sacha Inchi $222,4 \mathrm{Kcal} / 100 \mathrm{~g}$, y finalmente para las hamburguesas del tratamiento $20 \%$ de Sacha Inchi con $200,0 \mathrm{Kcal} / 100 \mathrm{~g}$.

El Sacha Inchi se considera un alimento energético por sus valores de grasa y calorías. A pesar de esto, se observa una disminución paulatina en los diferentes niveles de Sacha Inchi, esto debido a la perdida de ingredientes por cocción, en relación a la cantidad de grasa de cerdo que se utilizó en cada tratamiento.

En relación al análisis microbiológico, reportado en la Tabla 9, los resultados obtenidos para Coliformes Totales no sobrepasan los límites máximos permitidos por la norma INEN 2 346:2006. Tampoco se incumple la Norma Peruana, en varios casos no contemplados en la norma ecuatoriana. Se ha determinado ausencia de Escherichia coli, Stafilococcus aureus y Salmonella spp. Al respecto, Frazier, W y Westhoff, D. (1985), manifiestan que el Eschericha coli es un 
microorganismo relativamente sensible al calor, por lo que se puede destruir fácilmente a temperaturas de pasteurización o simplemente al cocinarse correctamente.

No existe referencia en las normas en lo referente a Mohos y Levaduras. Se ha demostrado que tanto en las carnes frescas y curadas, como en aves y mariscos frescos, se encuentran los siguientes géneros de bacterias: Achromobacter, Aeromonas, Aerobacter, Clostridium, Corynebacterium, Escherichia, Lactobacillus, Staphylocovvus y Streptococcus. Además se han aislado los siguientes géneros de hongos: Penicillium, Cladosporium, Mucor, Aspergillus, Alternaria, Fusarium y Rhizopus. También se encontraron las levaduras Cándida y Saccharomyces (Iparraguirre, M. 2009). No se encuentran microorganismos (Achromobacter, Clostridium, Proteus vulgarism, Pseudomonoas flurescens) que puedan alterar el sabor, olor y otras cualidades de la hamburguesa, que son causadas fundamentalmente por microorganismos proteolíticos y lipolíticos (Pelczar, R. y Reid, R. 1979). Según Larrañaga, I. (1999) los recuentos en carne picada suelen ser mayores que los de las correspondientes canales y su desarrollo dependerá de si el envasado se hace de forma aeróbica o anaeróbica. Además este tipo de carnes, una vez que se descongela, es al parecer más perecedero que la carne que no se congela.

Tabla 9. Resultados microbiológico de cuatro tipos de hamburguesas crudas elaboradas mediante el empleo de diferentes niveles de Sacha Inchi

\begin{tabular}{|c|c|c|c|c|c|c|}
\hline \multirow[b]{2}{*}{ Parámetro } & \multicolumn{4}{|c|}{$\begin{array}{c}\text { \% Sacha } \\
\text { Inchi }\end{array}$} & \multirow{2}{*}{$\begin{array}{c}\text { Límite } \\
\text { máximo } \\
\text { Norma } \\
\text { Peruana }\end{array}$} & \multirow{2}{*}{$\begin{array}{c}\text { Límite } \\
\text { máximo } \\
\text { Norma INEN }\end{array}$} \\
\hline & 0 & 10 & 15 & 20 & & \\
\hline $\begin{array}{l}\text { Recuento de mohos y } \\
\text { levaduras }\end{array}$ & $\begin{array}{c}2,7 x \\
10^{2}\end{array}$ & $\begin{array}{c}2,3 x \\
10^{2}\end{array}$ & $\begin{array}{c}2,1 \times \\
102\end{array}$ & $\begin{array}{c}1,4 \times \\
10^{2}\end{array}$ & - & - \\
\hline Coliformes totales & $\begin{array}{c}1,8 x \\
10^{2}\end{array}$ & $\begin{array}{c}1,6 x \\
10^{2}\end{array}$ & $\begin{array}{ll}1,5 & x \\
102 & \end{array}$ & $\begin{array}{c}1,4 x \\
10^{2}\end{array}$ & - & $2,4 \times 10^{3}$ \\
\hline Escherichia coli, UFC/g & $<10$ & $<10$ & $<10$ & $<10$ & 50 & $10^{3}$ \\
\hline $\begin{array}{l}\text { Stafilococcus aureus, } \\
\text { UFC/g }\end{array}$ & $<10$ & $<10$ & $<10$ & $<10$ & $10^{2}$ & $15 \times 10^{2}$ \\
\hline Salmonella spp. & - & - & 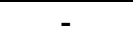 & - & Ausencia & Ausencia \\
\hline
\end{tabular}

Fuente: Norma Peruana (2008) Preparados de carnes refrigeradas o congeladas. Norma INEN (2006) Carne fresca y menudencias comestibles.

La aceptabilidad de las Hamburguesas elaboradas mediante diferentes niveles de Sacha Inchi presentó diferencias estadísticas $(\mathrm{P}<0,01)$, según Kruskal Wallis presentando medianas superiores en las hamburguesas de los tratamientos Control, 10 y $15 \%$ de pasta de Sacha Inchi con un valor de 4 puntos (Gusta un poco) y la menor mediana correspondió a las hamburguesas elaboradas con $20 \%$ de Sacha Inchi con un valor de 3 puntos (Ni gusta ni disgusta). 
Ramos, M. (2008) manifiesta que la grasa, principalmente la de cerdo, juega un papel importante en la determinación de las propiedades organolépticas de los productos cárnicos. Ranken, M. (1993) describe que en algunos alimentos, entre ellos la carne, las grasas son responsables de parte del aroma, contribuyendo así a la palatabilidad de la dieta. Por esta razón la disminución de la grasa de cerdo y el aumento de la pasta de Sacha Inchi, puede haber ocasionado una menor aceptabilidad de varios panelistas para los tratamientos 15 y $20 \%$ de Sacha Inchi. En la industria de alimentos, los sustitutos de grasa están constituidos por una gran variedad de compuestos que se utilizan para reemplazar total o parcialmente la grasa empleada en la elaboración de hamburguesas manteniendo muchas de las características de textura y sabor, que los hacen apetecibles por los consumidores (Gershoff, S. 1995).

En lo referente a la intención de compra, las hamburguesas elaboradas con $10 \%$ de Plukenetia volubilis presentaron el $71,67 \%$ de respuesta positiva, es decir los panelistas si comprarían la hamburguesa, mientras que un $20 \%$ no la comprarían, para el tratamiento $15 \%$ de Sacha Inchi en las hamburguesas, el 70,00 \% si comprarían la hamburguesa, y el 23,33\% no lo harían, en cuanto a las hamburguesas del tratamiento Control el $61,67 \%$ de los encuestados si la comprarían, y un $26,67 \%$ no lo harían, y por último las hamburguesas del tratamiento $20 \%$ de Plukenetia volubilis un 55,00 \% de personas encuestadas si la comprarían, mientras que el 35,00 $\%$ no comprarían las hamburguesas.

Analizando a la composición de ácidos grasos en la hamburguesa elaborada con la utilización del $10 \%$ de Plukenetia volubilis es importante resaltar el Omega 3 con $16.82 \%$, el Omega 6 con $21.97 \%$ y el Omega 9 con $34.88 \%$, que se ven reflejados en los valores determinados en la constitución del Sacha Inchi. También existe un incremento de Ácido Palmítico, Esteárico y Oleico, aportados por la composición de la carne y grasa (García, J. 2008).

Los resultados del análisis económico se muestran en la Tabla 10. Obsérvese que se consideraron los costos necesarios para la obtención de $10 \mathrm{~kg}$ de hamburguesas de los diferentes tratamientos, así como los ingresos obtenidos por la cotización del producto terminado.

Según los cálculos, se ha determinado el mayor índice de Beneficio/Costo en las hamburguesas pertenecientes al tratamiento de $10 \%$ de Plukenetia volubilis, reportándose un índice de 1,25 USD, lo cual indica que por cada dólar invertido durante el ensayo se obtiene una rentabilidad de 0,25 USD. Los demás tratamientos presentaron indicadores inferiores, ya sea por la inclusión de niveles superiores de pasta de Sacha Inchi o por una menor cotización en el producto terminado en el caso del tratamiento testigo ya que no es considerado como tal un alimento funcional. 
Tabla 10. Evaluación económica de los cuatro tipos de hamburguesas

\begin{tabular}{|c|c|c|c|c|}
\hline \multirow{2}{*}{$\begin{array}{l}\text { Concepto } \\
\text { EGRESOS }\end{array}$} & \multicolumn{3}{|c|}{ Niveles de Sacha inchi (\%) } & \multirow[b]{2}{*}{20} \\
\hline & 0 & 10 & 15 & \\
\hline Carne de Res $^{\top}$ & 27,72 & 27,72 & 27,72 & 27,72 \\
\hline Grasa de Cerdo $^{2}$ & 2,00 & 1,00 & 0,50 & 0,00 \\
\hline Pasta de Sacha inchi ${ }^{3}$ & 0,00 & 2,50 & 3,75 & 5,00 \\
\hline Harina de Trigo $^{4}$ & 2,00 & 2,00 & 2,00 & 2,00 \\
\hline Condimentos $^{5}$ & 1,22 & 1,22 & 1,22 & 1,22 \\
\hline Aditivos $^{6}$ & 0,59 & 0,59 & 0,59 & 0,59 \\
\hline Mano de Obra' & 5,00 & 5,00 & 5,00 & 5,00 \\
\hline Subtotal Egresos & 38,53 & 40,03 & 40,78 & 41,53 \\
\hline \multicolumn{5}{|l|}{ INGRESOS } \\
\hline $\begin{array}{l}\text { Cotización de } \\
\text { Hamburguesas }^{8}\end{array}$ & 40,0 & 50,0 & 50,0 & 50,0 \\
\hline Subtotal Ingresos & 40,00 & 50,00 & 50,00 & 50,00 \\
\hline Beneficio / Costo & 1,04 & 1,25 & 1,23 & 1,20 \\
\hline
\end{tabular}

1: $\$ 3,96 / \mathrm{Kg}$ de Carne. $\quad 5: \$ 1,22 /$ Tratamiento/Condimentos

2: $\$ 1,00 / \mathrm{Kg}$ de Grasa $\quad 6: \$ 0,59 /$ Tratamiento/Aditivos

3: $\$ 3,00 / \mathrm{Kg}$ Pasta de Sacha inchi $\quad$ 7: $\$ 5 /$ Tratamiento/Mano de Obra

4: $\$ 2,00 / \mathrm{Kg}$ de Harina de Trigo $\quad 8: \$ 4,00 / \mathrm{Kg}$ de Hamburguesa $\mathrm{R} ; 5,00 / \mathrm{Kg}$ de Hamburguesa SI

\section{Conclusiones}

1. Se determinó que mediante la utilización Plukenetia volubilis (Sacha Inchi) en un $10 \%$ en remplazo al peso en grasa para la elaboración de hamburguesas, se obtiene un mayor porcentaje de proteína $(20,86 \%)$ y disminución de grasa $(12,91 \%)$.

2. La carga microbiológica encontrada en cuanto a Mohos y Levaduras y Coliformes Totales, disminuyen con el empleo de Sacha Inchi, lo cual ubica al producto dentro de las normas ecuatorianas (INEN), para la industrialización de este producto.

3. La hamburguesa del tratamiento $10 \%$ de Sacha Inchi, obtuvo los mayores indicadores en aceptabilidad e intención de compra del producto, por lo tanto es la más aceptada.

4. La hamburguesa del tratamiento $10 \%$ de Sacha Inchi, presenta valores de Omega-3 de 16.82 $\%$, de Omega-6 $21.97 \%$ y de Omega-9 $34.88 \%$, y un alto nivel proteico, obteniéndose un alimento funcional, que podría disminuir los riesgos de enfermedades cardiovasculares en los consumidores.

5. La mayor rentabilidad se alcanzó al utilizar el $10 \%$ de Sacha Inchi en la elaboración de hamburguesas al determinarse un índice de Beneficio/Costo de 1.25 USD. 


\section{Bibliografía}

Anaya (2006) (http://www.agenciaperu.com/reportes/2006/jul/sacha_inchi.html)

FRAZIER, W y WESTHOFF, D. Microbiología de los alimentos. Zaragoza-España. Editorial Acribia, S. A., 1985., Pp. 356-359.

GARCÍA, H. (1992) Estudio de Sacha Inchi. Lima-Perú. Conferencias de investigaciones apoyadas por Fundeagro 1988 - 1992. Tomo I. Proyecto de trasformación de la tecnología agropecuaria. Pp. 61-63.

GARCÍA, J. (2008) Procesamiento y Preservación de la Carne I. Riobamba-Ecuador. Compendio. Maestría en Industrias Pecuarias ESPOCH. Facultad de Ciencias Pecuarias. Profesor de la Universidad Autónoma de Chihuahua México. Facultad de Zootecnia. Pp. 56-72.

GERSHOFF, S. (1995) Nutrition evaluation of dietary fat substitutes. Washington-Estados Unidos. Nutrition Reviews. Vol. 53, No. 11. Pp. 11-12.

HAMAKER, E. y otros. (1992) Aminoacid and Fatty Acid Profil of the Inca Peanut (Plukenetia volubilis. L). lowa-Estados Unidos. En: American association of cereal chemist. Note. Vol.69. No. 4. Pp. 461-465.

HÁZEN, S. y STOVESAND, P. (1980) Agroindustrias Amazónicas. Washington-Estados Unidos. Editorial Amazon. Pp. 89.

INSTITUTO ECUATORIANO DE NORMALIZACIÓN (INEN). (2006) Quito-Ecuador. Carne fresca y menudencias comestibles. Norma técnica ecuatoriana NTE INEN 2346.

LARRAÑAGA, I. y otros. (1999) Control e higiene de alimentos. Barcelona-España. Editorial Cobra S. L. Pp. 323.

MELGAREJO, I. y MAURY, M. (2002) Elaboración de Hamburguesa a partir de Prochylodus nigricans "BOQUICHICO". Iquitos-Perú. En: Revista Amazónica de Investigación Alimentaria, Facultad de Ingeniería en Industrias Alimentaria, UNAP, Vol. 2, № 1. Pp. 79-87.

OBREGÓN, L. (1993) "Obtención de sacha inchi (Plukenetia volubilis) en polvo secado por atomización". Facultad de Agronomía, Universidad Nacional Autónoma La Molina. Lima-Perú. Tesis de Maestría.

ORGANIZACIÓN MUNDIAL DE LA SALUD (OMS). (2009) Buenos Aires-Argentina. Cumbre, Qué está haciendo la OMS para ayudar a los países a mejor la inocuidad de los alimentos.

Pascual, G. y Mejía M. (2000) Extracción y caracterización de aceite de sacha inchi (Plukenetia Volubilis L). Anales Científicos UNALM. 42:143-57 
PELCZAR, R. y REID, R. (1979) Microbiología. La Habana-Cuba. Editorial Pueblo y Educación. Pp. 476-478.

RAMOS, M. (2008) Características Físicas y Químicas de la Carne, Cambios que Ocurren Durante Su Maduración, Microbiología y Conservación de la Carne. Riobamba-Ecuador. Compendio, Maestría en Industrias Pecuarias ESPOCH, Facultad de Ciencias Pecuarias. Profesor de la Universidad Técnica de Ambato. Facultad de Ingeniería en Alimentos. Pp. 58-63.

RANKEN, M. (1993) Manual de industrias de los alimentos. Zaragoza-España. 2da edición., editorial Acribia S. A. Pp. 36-37.

SACHA INCHI. (2009/02/12) http://www.omegagelcaps.com/spanish/característica.html

SALAZAR, D. (2008) Productos Cárnicos. Ambato-Ecuador. Conferencia, Universidad Técnica de Ambato, Facultad de Ingeniería en Alimentos. Pp. 14-20.

VÁZQUEZ, M. (2005) Alimentación y nutrición: Manual teórico-práctico. Quito-Ecuador. Editorial Díaz de Santos. ISBN 84-7978-715-5. Pp. 46-48.

Velázquez, F., Julio César Vargas, Verónica Andrade, Daniela Baldeón, Janeth Sánchez. (2014) Plukenetia volubilis L. (SACHA INCHI - TICASU) riqueza natural para la salud humana y valor agregado en las carnes del cerdo criollo ecuatoriano en la Amazonia. HUELLAS DEL SUMACO - Revista Socio Ambiental de la Amazonía ecuatoriana. Universidad Estatal Amazónica. VOLUMEN 11. ISSN: 1390-6801.

YÁNEZ, E. y BIOLLEY. E. (1999) Sustitutos de grasas en la alimentación humana. MedellínColombia. Archivos Latinoamericanos de Nutrición. Vol. 49, № 2. Pp. 101-103. 